\title{
Length Contraction with Speed Explained as a Relativistic Doppler Effect Constantin SANDU ${ }^{1}$, Dan BRASOVEANU ${ }^{2}$ \\ ${ }^{1}$ Romanian Research and Development Institute for Gas Turbines-COMOTI, 220D, Iuliu Maniu Bvd., Sector 6, OP76, CP174, Bucharest, Romania \\ constantin.sandu@comoti.ro \\ ${ }^{2}$ Systems Engineering Group Inc. (SEG), USA \\ 9861 Broken Land Pkwy, suite 350, Columbia, MD, 21046, USA \\ brasovdx@yahoo.com
}

\section{ABSTRACT}

In this paper the authors show that the 'mechanism' of length contraction is simple and can be explained by the wave feature of body micro-components.

From this point of view, the length contraction with speed perceived by a fixed observer is a Doppler relativistic effect.

\section{Indexing terms/Keywords}

length contraction with speed, relativistic contraction

\section{Academic Discipline And Sub-Disciplines}

Physics, Relativity, Special Theory of Relativity

\section{SUBJECT CLASSIFICATION}

83A05

\section{TYPE (METHOD/APPROACH)}

\author{
Theoretical
}

\section{INTRODUCTION}

The Special Theory of Relativity (STR) states that the length of a body contracts with speed. After over 100 years of relativity, the contraction of length is still considered a weird thing because this phenomenon did not yet received an intuitive demonstration and practically it was not observed.

The phenomenon can be easily accepted by intuition if the wave feature of micro components of body is considered. Everybody easily understands the Doppler effect for sound sources: The frequency of sound heard by an observer increases when the sound source moves towards observer and decreases when the sound source departs by observer. Similar, the frequency of associated waves of micro-components of a body are subject to the Doppler relativistic effect when are perceived by an external observer, i.e. the frequency of wave (of micro-component) which moves towards the observer is perceived higher than the frequency of the same wave (of micro-component) when it departs by observer. But the observer sees only an average frequency and an average wavelength of the two cases. This average wavelength is contracted with the factor $\sqrt{1-V^{2} / c^{2}}$ relatively to the situation when the micro-component is at rest because the terms which contain speed at the power of 1 disappear. Thus, the whole length of body (which is a sum of average wave lengths of body micro-components) contracts with the factor $\sqrt{1-V^{2} / c^{2}}$.

\section{DEMONSTRATION}

For simplicity, assume that the matrix of body is composed of a number $n$ of fermions, $f$, connected by $n-1$ bosons, $b$, aligned with the fermions along of an axis. In the matrix of body all the fermions and bosons can be associated with stationary waves between fixed positions A, B as presented in Figure 1.

For a fixed observer, the total length $L_{0}$ of such a body when it is at rest (speed $V=0$ ) is:

$\mathrm{L}_{0}=\mathrm{n} \cdot \lambda_{\mathrm{Of}}(\mathrm{n}-1) \cdot \lambda_{\mathrm{Ob}}$

where $\lambda_{0 f}$ and $\lambda_{0 b}$ are the wavelengths of associated waves of fermions and bosons respectively when the body is at rest.

If the body begins to move with speed $\mathrm{V} \neq 0$ to the observer, the observer will note a shift in the frequency of the associated waves of fermions and bosons caused by the relativistic Doppler effect [1]. 


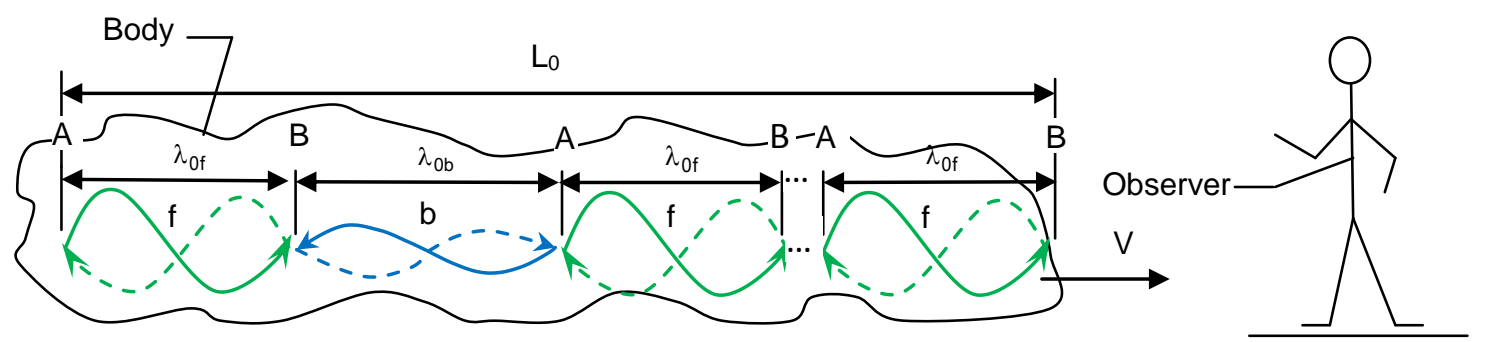

Fig.1:The Relativistic Doppler Effect explains why length decreases with speed

When the associated waves of fermions and bosons propagate to the right (to the observer), case 1', the observed frequencies of associated waves for fermions and bosons are higher:

$v_{1^{\prime} f, b}=v_{0 f, b} \frac{1+\frac{V}{c}}{\sqrt{1-\frac{V^{2}}{c^{2}}}}$

Vice-versa, when the wave is moving away from the observer (case 1"), the observer sees smaller frequencies:

$v_{1 " f, b}=v_{0 f, b} \frac{1-\frac{V}{c}}{\sqrt{1-\frac{V^{2}}{c^{2}}}}$

The average frequencies of associated waves of fermions and bosons seen by observer are obviously

$v_{11 ' f f, b}=\frac{v_{0 f, b}}{\sqrt{1-\frac{v^{2}}{c^{2}}}}$

The average periods are

$T_{1^{\prime} 1^{\prime \prime}, \mathrm{b}}=\frac{\sqrt{1-\frac{\mathrm{V}^{2}}{\mathrm{c}^{2}}}}{v_{0 f, \mathrm{~b}}}$

The average wavelengths are:

$\lambda_{1^{\prime} 1^{\prime \prime}, \mathrm{b}}=\mathrm{c} \cdot \mathrm{T}_{1^{\prime} 1^{\prime \prime} \mathrm{f}, \mathrm{b}}=\mathrm{c} \cdot \frac{\sqrt{1-\frac{\mathrm{V}^{2}}{\mathrm{c}^{2}}}}{v_{\mathrm{Of}, \mathrm{b}}}=\lambda_{\mathrm{Of}, \mathrm{b}} \cdot \sqrt{1-\frac{\mathrm{V}^{2}}{\mathrm{c}^{2}}}$

And the total length becomes:

$\mathrm{L}_{1^{\prime} 1^{\prime \prime}}=\mathrm{n} \cdot \lambda_{1^{\prime} 1^{\prime \prime f}}(\mathrm{n}-1) \cdot \lambda_{1^{\prime} 1^{\prime \prime b}}=\sqrt{1-\frac{\mathrm{V}^{2}}{\mathrm{c}^{2}}}\left[\mathrm{n} \cdot \lambda_{\mathrm{Of}}+(\mathrm{n}-1) \cdot \lambda_{\mathrm{ob}}\right]=\mathrm{L}_{0} \cdot \sqrt{1-\frac{\mathrm{V}^{2}}{\mathrm{c}^{2}}}$

So, the observer sees that the length of body is contracted and this contraction is real, i.e., if the observer will make a photograph of that moving body, the dimension of moving body in the direction of movement will be smaller than in the case when body is at rest.

\section{CONCLUSIONS}

This paper demonstrates that contraction of bodies with speed can be seen as a relativistic Doppler effect.

According to the Standard Model, a body is composed only by fermions and bosons. If these fermions and bosons are seen as stationary waves, the average wave length of fermions and bosons decreases with speed with the factor $\sqrt{1-\mathrm{V}^{2} / \mathrm{c}^{2}}$.

This paper gives only a physical explanation for a better understanding of length contraction with speed showing that contraction is a natural effect which must appear when a body moves relatively to an observer.

The contraction of body is real i.e., in a photograph made by a fixed observer the dimension of that body will be contracted on direction of speed. 


\section{ACKNOWLEDGMENTS}

This represents original research belonging to authors

\section{REFERENCES}

1. Einstein , A., June 30/1905 - Electrodynamics of Moving Bodies, page 16, Annalen der Physik.

\section{Author' biography with Photo}

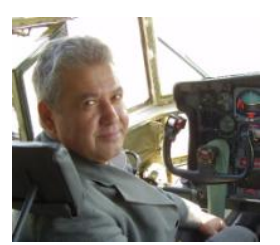

Aerospace engineer, Romanian scientist. Scientific researcher at Romanian Research and Development Institute for Gas Turbines-COMOTI, Bucharest, Romania. PhD in power transmission through mechanical vibrations. Over 35 papers in the field of physics of flight and aerospace propulsion. Project manager in 5 national and European research projects, 8 inventions.

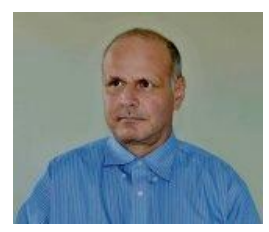

Dr. Dan Brasoveanu is an expert in theoretical and applied physics including physics of propulsion, guidance navigation and control (GNC). He is also an expert in satellite sensors and nuclear detection systems. He worked for NASA, USA Department of Defense and Department of Homeland Security. He is the author or co-author of more than 40 science papers on propulsion, satellite GNC and theoretical physics published in science journals and refereed conference publications and of a book on quantum and relativistic mechanics included in the library of Conseil Européen pour la Recherche Nucléaire (CERN). 